\title{
Actualización: Osteoporosis primaria: evaluación diagnóstica y estratificación del riesgo de fractura para la toma de decisiones clínicas
}

\author{
Primary osteoporosis: diagnostic evaluation and risk stratification of fracture for clinical decision-making
} Vilda Discacciati*

Resumen
Las fracturas osteoporóticas son una causa frecuente de morbilidad en la población de edad avanzada. El adecuado ras-
treo y manejo de uno de sus principales factores asociados, la osteoporosis primaria (OP), constituye un desafío importante
para el médico de atención primaria. En la presente revisión se consideran los principales elementos implicados en el diag-
nóstico de la OP, haciendo hincapié en el uso de modelos de predicción de riesgo de fractura que toman a la densitometría
mineral ósea como un elemento más de la valoración, para considerar entonces que pacientes podrían beneficiarse de
tratamientos farmacológicos.

\section{Abstract}

Osteoporotic fractures are a common cause of morbidity in the elderly population. Proper screening and management of primary osteoporosis (PO) is a major challenge for the primary care physician. In this review are considered bone mineral density as part of the evaluation. The author analyze the key elements involved in PO diagnosis, with emphasis on the use of models for predicting fracture risk, to consider patients who could benefit from medications.

Palabras clave: Osteoporosis, diagnóstico, tratamiento, toma decisión. Key words: Osteoporosis, diagnosis, treatment, decision making.

Disacacciati V. Osteoporosis: evaluación diagnóstica y estratificación del riesgo de fractura para la toma de decisión Evid. actual. práct. ambul; 11(2):49-52, Mar-Abr.2008

\section{Introducción}

En las últimas décadas ha sido muy extensa la cantidad de información publicada sobre osteoporosis ${ }^{1}$ proveniente de artículos médicos y dirigida a la comunidad. Se ha progresado en el conocimiento de la entidad, de su impacto clínico, de sus costos sanitarios, y se han extendido las posibilidades diagnósticas y terapéuticas. Así, la osteoporosis pasó de ser una entidad con indicaciones de rastreo cuestionable, a formar parte de las enfermedades que tienen recomendaciones específicas de serlo.

La elevada prevalencia de osteoporosis y osteopenia en las mujeres mayores de 50 años, convierten a estas problemáticas en temas relevante para la salud pública. Su importancia se magnifica a medida que la población envejece, dado el incremento en la incidencia de fracturas osteoporóticas ${ }^{2}$. Sin embargo y como sucede con otras entidades clínicas, diferentes sectores sociales le atribuyen distinta importancia. Por ejemplo, desde el punto de vista de las pacientes, su significado no será el mismo para quienes inician naturalmente su menopausia, que para las mujeres más añosas; mientras que para los profesionales de la salud, también tendrá diferente implicancia según su especialidad, su sesgo de formación profesional y el ámbito social donde le toque desempeñarse.

El riesgo anual de fractura osteoporótica depende de la edad de la paciente. La incidencia es de una fractura cada dos mil mujeres antes de los 60 años, de una a dos a los 65 años, y de seis a siete a los 75 años. Por otro lado, la información disponible sugiere que a través del tratamiento farmacológico se logra una importante reducción en el riesgo de estas fracturas (30 a 50\%). Sin embargo, la demostración de estos beneficios atribuibles al tratamiento* ha requerido la realización de estudios prolongados (por lo menos tres a cinco años) y con gran número de personas observadas; y ha conducido discusiones sobre la seguridad de estos tratamientos farmacológicos, fundamentalmente en aquellos grupos con bajo riesgo absoluto $^{3}$ de padecer fracturas osteoporóticas.

\section{Evaluación diagnóstica}

En su consenso de 2006, la Sociedad Norteamericana de Menopausia' (en inglés NAMS) propone la prevención de fracturas como el objetivo primordial del tratamiento de la osteoporosis ${ }^{4}$. Otras metas implicadas incluyen: retrasar la pérdida de masa ósea -manteniendo su fortaleza- y minimizar los fac- tores que contribuyen a la ocurrencia de fracturas. Según este organismo, la evaluación de las pacientes debe ser completa, incluyendo antecedentes clínicos, examen físico y estudios diagnósticos. Enfatizan que los principales factores asociados a la pérdida de masa ósea son el avance de la edad, los factores genéticos, los hábitos o estilo de vida (alimentación, tabaquismo y sedentarismo) la delgadez y la menopausia; y recomiendan que el manejo debería focalizarse en las medidas no farmacológicas como la promoción de una dieta que contenga suficiente calcio y vitamina $\mathrm{D}$, y de la práctica de actividad física'.

Haciendo referencia a este último punto, resulta importante resaltar que en los últimos años se ha facilitado el acceso a la densitometría mineral ósea (DMO) por lo que se ha agregado una herramienta para la toma de decisiones médicas. La DMO predice riesgo de fractura y en combinación con otros factores, puede estimar el riesgo absoluto de fracturas para la mujer posmenopáusica de raza blanca -dado que en este punto existen diferencias étnicas ${ }^{5}$, derivadas de diferencias genéticas y ambientales como los hábitos nutricionales, el nivel de actividad física, la exposición al sol, etc.

Hasta el momento, la información proveniente de revisiones sistemáticas y de estudios de costo-efectividad sugiere evaluar con una DMO a las mujeres posmenopáusicas a partir de los 65 años. Sin embargo, dada el bajo valor predictivo positivo DMO en la predicción del riesgo de fracturas (ver tabla 1) pueden sobrevenir dos problemas al utilizar en forma aislada este método para la valoración de las pacientes con osteoporosis ya que:

1) Habrá pacientes cuya condición no será detectada y por lo tanto, no serán "adecuadamente" tratadas.

2) Serán "sobre-tratadas" aquellas pacientes con bajo riesgo global de fractura y que sólo deberían recibir recomendaciones focalizadas en la promoción de hábitos saludables.

Por lo tanto, arribamos a una primera conclusión: que la medición de la densidad mineral ósea no debería ser el único dato determinante de las decisiones médicas.

Tabla 1: valor predictivo positivo para fracturas osteoporóticas en la comunidad de Madrid (España) de la medición de la densidad mineral ósea de acuerdo a la edad de la paciente.

\begin{tabular}{c|c}
\hline Edad & Valor predictivo positivo \\
\hline $\mathbf{6 0}$ años & aproximadamente $5 \%$ \\
\hline $\mathbf{7 0}$ años & $9 \%$ \\
\hline $\mathbf{8 0}$ años & $36 \%$ \\
\hline
\end{tabular}

Servicio de Medicina Familiar y Comunitaria. Hospital Italiano de Buenos Aires. vilda.discacciati@gmail.com 
Considerando los posibles efectos adversos y costos de los tratamientos farmacológicos, en los últimos tiempos y con el objetivo de determinar a quienes evaluar con una DMO y a quiénes tratar, ha cobrado relevancia la estratificación del riesgo de fractura. Así, diversos investigadores y grupos de trabajo han desarrollado reglas de predicción clínica a través de la combinación y ponderación de los diferentes factores asociados a osteoporosis y al riesgo de fractura ${ }^{678}$. Destacaremos en particular el documento elaborado por el Grupo de Trabajo en Osteoporosis de la Comunidad de Madrid ${ }^{9}$. En primer lugar, esta guía propone estimar el riesgo de fractura considerando los siguientes cuatro elementos predictores:
1. La variabilidad geográfica ya que, como muestra la tabla 2 , existen diferencias regionales en el riesgo de fractura.

2. La edad de la paciente, ya que la incidencia de fractura de cadera aumenta marcadamente a partir de los 75 a 79 años de edad.

3. Los factores de riesgo clínico, principalmente el antecedente familiar de fractura, el antecedente personal de fractura periférica después de los 50 años o de fractura vertebral previa y un índice de masa corporal menor a $19 \mathrm{~kg} / \mathrm{m}^{2} ; \mathrm{y}$ secundariamente el consumo de tabaco y/o alcohol.

4. La densidad mineral ósea, que interviene como un factor complementario ya que da información sobre el riesgo de fractura.

Tabla 2: riesgo relativo y probabilidad (riesgo absoluto) a diez años de fractura de cadera en hombres y mujeres de acuerdo a su edad y la región geográfica en la que viven. Se aclara que en la mayoría las publicaciones revisadas por Kanis y col..$^{10}$, la información es regional y puede no ser extrapolable al resto del país en cuestión.

\begin{tabular}{|c|c|c|c|c|c|c|c|c|c|c|}
\hline \multirow[t]{2}{*}{ Riesgo } & \multirow[t]{2}{*}{ Región con reportes } & \multirow{2}{*}{$\begin{array}{l}\text { Riesgo } \\
\text { relativo }\end{array}$} & \multicolumn{4}{|c|}{ Riesgo absoluto en 10 años en Hombres } & \multicolumn{4}{|c|}{ Riesgo absoluto en 10 aî̃os en Mujeres } \\
\hline & & & 50 años & 60 años & 70 años & 80 años & 50 años & 60 años & 70 años & 80 años \\
\hline \multirow[t]{4}{*}{ Bajo } & Chile & 0,08 & \multirow[t]{2}{*}{ ND } & $0,1 \%$ & $0,3 \%$ & \multirow[t]{3}{*}{ ND } & \multirow[t]{2}{*}{ ND } & $0,2 \%$ & $0,6 \%$ & \multirow[t]{3}{*}{ ND } \\
\hline & Venezuela & 0,17 & & $0,2 \%$ & $0,8 \%$ & & & $0,3 \%$ & $1,5 \%$ & \\
\hline & Korea & 0,18 & $0,2 \%$ & $0,3 \%$ & $0,5 \%$ & & $0,1 \%$ & $0,2 \%$ & $0,5 \%$ & \\
\hline & Turquía & 0,18 & $0,2 \%$ & $0,5 \%$ & $0,6 \%$ & $1,5 \%$ & $0,1 \%$ & $0,4 \%$ & $0,4 \%$ & $0,1 \%$ \\
\hline \multirow[t]{4}{*}{ Intermedio } & China (Beijing) & 0,29 & $0,3 \%$ & $0,4 \%$ & $1,4 \%$ & $1,6 \%$ & $0,2 \%$ & $0,7 \%$ & $1,1 \%$ & $1,6 \%$ \\
\hline & Argentina (La Plata) & 0,36 & $0,1 \%$ & $0,4 \%$ & $1,5 \%$ & ND & $0,2 \%$ & $0,7 \%$ & $4,8 \%$ & ND \\
\hline & España (Barcelona) & 0,39 & $0,1 \%$ & $0,5 \%$ & $1,6 \%$ & $4,2 \%$ & $0,2 \%$ & $0,8 \%$ & $3,3 \%$ & 9,75 \\
\hline & Japón & 0,39 & $0,2 \%$ & $0,5 \%$ & $1,3 \%$ & $3,3 \%$ & $0,2 \%$ & $0,8 \%$ & $3,2 \%$ & $9,6 \%$ \\
\hline \multirow[t]{2}{*}{ Alto } & Italia (Génova) & 0,61 & $0,4 \%$ & $0,7 \%$ & $1,4 \%$ & $2,5 \%$ & $0,5 \%$ & $1,4 \%$ & $5,2 \%$ & $14 \%$ \\
\hline & Portugal (Acores) & 0,57 & $0,5 \%$ & $0,9 \%$ & $1,9 \%$ & $2,6 \%$ & $0,3 \%$ & $1,2 \%$ & $5,3 \%$ & $6,7 \%$ \\
\hline \multirow[t]{2}{*}{ Muy alto } & EE.UU. (Minessota) & 0,78 & $0,4 \%$ & $1,1 \%$ & $2,1 \%$ & $6,1 \%$ & $0,7 \%$ & $1,8 \%$ & $5 \%$ & $14,2 \%$ \\
\hline & Suecia & 1 & $0,5 \%$ & $1,5 \%$ & $3,9 \%$ & $9,1 \%$ & $0,6 \%$ & $2,2 \%$ & $7,1 \%$ & $17,7 \%$ \\
\hline
\end{tabular}

Resumido de: Kanis y col. International variations in hip fracture probabilities: implications for risk assessment. J Bone Miner Res. 2002; 17:1237-44. ND: no disponible. Las comparaciones se han realizado tomando como referencia al riesgo de la población de Suecia, a la que se le asignó arbitrariamente un riesgo relativo de 1 .

Figura 1: Variabilidad geográfica en la probabilidad de fracturas

Noruega
Suecia
EE.UU. (Minessota)
Italia (Génova)
Portugal (Acores)
Japón
Regiones de España (Barcelona)
Argentina (La Plata)
China (Beijing)
Turquía
Korea
Venezuela
Chile

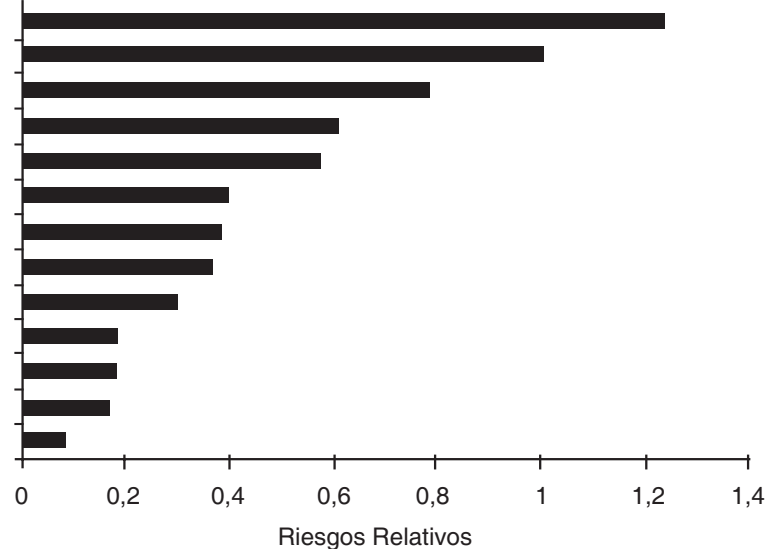

Construida sobre la base de la información reportada por: Kanis y col. International variations in hip fracture probabilities: implications for risk assessment. J Bone Miner Res. 2002; 17:1237-44. Las comparaciones se han realizado tomando como referencia al riesgo de la población de Suecia, a la que se le asignó arbitrariamente un riesgo relativo de 1.

Luego, establece como principal elemento para la toma de decisiones a la valoración del riesgo absoluto de fractura durante los siguientes diez años (de acuerdo a las estimaciones españolas). Este riesgo absoluto surge de una regla de predicción que toma en cuenta la edad y las variables clínicas con mayor capacidad predictiva del riesgo de fractura de cadera. Ver tablas 3 y 4.
Tabla 3: peso de cada variable predictora.

\begin{tabular}{l|l|l}
\hline \multicolumn{2}{|c|}{ Variable predoctora } & Puntos \\
\hline \multirow{2}{*}{ Indice de masa corporal menor a 19kg/m2 } & 1 \\
\hline \multirow{2}{*}{ Antecedente } & personal de fractura luego de los $\mathbf{5 0}$ años & 1 \\
\cline { 2 - 3 } & de fractura de cadera en madre, padre 0 hermana & 1 \\
\hline Fractura vertebral morfométrica (con disminución de $\mathbf{2 0} \%$ de la altura vertebral) & 2 \\
\hline
\end{tabular}


Tabla 4: riesgo absoluto a diez años en población española de fractura de cadera $(C)$ y de fractura vertebral morfométrica $(\mathrm{V})$ teniendo en cuenta la edad y otros antecedentes valorados clínicamente de acuerdo al escore de la tabla 3.

\begin{tabular}{|c|c|c|c|c|c|c|c|c|c|c|c|c|}
\hline \multicolumn{13}{|c|}{ Puntuación cliníca de riesgo } \\
\hline \multirow{2}{*}{$\begin{array}{c}\text { Edad en } \\
\text { aî́os }\end{array}$} & \multicolumn{2}{|c|}{$\mathbf{0}$} & \multicolumn{2}{|c|}{1} & \multicolumn{2}{|c|}{2} & \multicolumn{2}{|c|}{3} & \multicolumn{2}{|c|}{4} & \multicolumn{2}{|c|}{5} \\
\hline & C & v & E & V & E & v & C & V & C & v & C & V \\
\hline 50 & $0,2 \%$ & $0,9 \%$ & $0,5 \%$ & $1,8 \%$ & $1,0 \%$ & $3,6 \%$ & $1,5 \%$ & $5,4 \%$ & $2,0 \%$ & $7,1 \%$ & $2,5 \%$ & 8,85 \\
\hline 60 & $1,0 \%$ & $3,3 \%$ & $2,1 \%$ & $6,5 \%$ & $4,1 \%$ & $12,5 \%$ & $6,1 \%$ & $18,1 \%$ & $8,0 \%$ & $23,3 \%$ & $9,9 \%$ & $28,1 \%$ \\
\hline 70 & $3,0 \%$ & $4,7 \%$ & $5,8 \%$ & $9,2 \%$ & $11,3 \%$ & $17,4 \%$ & $16,4 \%$ & $24,7 \%$ & $21,1 \%$ & $31,1 \%$ & $25,5 \%$ & $36,4 \%$ \\
\hline 80 & $5,3 \%$ & $4,2 \%$ & $10,2 \%$ & $8,1 \%$ & $19,1 \%$ & $15,4 \%$ & $20,5 \%$ & $21,8 \%$ & $25,5 \%$ & $27,4 \%$ & $37,4 \%$ & $32,1 \%$ \\
\hline
\end{tabular}

Modificado de: Dirección General de Farmacia y Productos Sanitarios. Recomendaciones para la valoración y tratamiento d e la osteoporosis primaria en mujeres de la Comunidad de Madrid. Madrid: Comunidad de Madrid, Consejería de Sanidad; 2007.

Finalmente y teniendo en cuenta estos parámetros, se establecen recomendaciones sobre la indicación de la DMO y el tratamiento farmacológico según el riesgo individual de fractura osteoporótica. Ver cuadro 5. Vale aclarar que estas recomendaciones son para la generalidad de la población, ya que los autores sugieren que las pacientes que presenten valores extremos de los factores de riesgo, por ejemplo múltiples fracturas después de los 50 años, un índice de masa corporal de 15 en mujeres mayores de 70 años o la documentación de fracturas vertebrales múltiples, sean evaluadas en forma individual y no sobre la base de esta herramienta.

Tabla 5: herramienta para la toma decisiones diagnósticas y terapéuticas para la prevención de fracturas osteoporóticas en mujeres de acuerdo a su edad y nivel de riesgo.

\begin{tabular}{|c|c|c|}
\hline $\begin{array}{l}\text { Edad } \\
\text { en años }\end{array}$ & $\begin{array}{c}\text { Puntuación } \\
\text { de riesgo }\end{array}$ & Toma de decisiones \\
\hline \multirow{3}{*}{$\begin{array}{r}\text { Mayores } \\
\text { de } 75^{\mathrm{a}}\end{array}$} & 0 & Ni tratamiento ni densitometría \\
\hline & 1 y 2 & Densitometría Tratamiento si el escore $\mathrm{T}$ es menor a $-2,5$ \\
\hline & 3 a 5 & Tratamiento directamente \\
\hline \multirow[t]{3}{*}{60 a 75} & 0 y 1 & Ni tratamiento ni densitometría \\
\hline & 2 y 3 & Densitometría| Tratamiento si el escore $\mathrm{T}$ es menor a $-2,5$ \\
\hline & 4 y 5 & Tratamiento directamente \\
\hline \multicolumn{2}{|c|}{ Menores de 60} & Ni tratamiento ni densitometría \\
\hline
\end{tabular}

Construida sobre la base del algoritmo de toma de decisiones recomendado por: Dirección General de Farmacia y Productos Sanitarios.

Recomendaciones para la valoración y tratamiento de la osteoporosis primaria en mujeres de la Comunidad de Madrid. Madrid:

Comunidad de Madrid, Consejería de Sanidad; 2007. aSe aclara que no han sido incluidas mujeres mayores de 80 años en los ensayos clínicos que evaluaron la eficacia de los fármacos.

\section{Conclusiones}

A pesar de que en la práctica clínica habitual y según los criterios de clasificación de la Organización Mundial de la Salud (OMS) de 1994, la osteoporosis se diagnostica "de manera densitométrica", la DMO sólo proporciona información sobre uno de los factores de riesgo de fractura, dependiendo su valor predictivo del riesgo basal (probabilidad pre-test) de fractura. En este contexto, los modelos de ponderación de riesgo como el esquematizado en la presente actualización, intentan identificar a aquellas mujeres que más se beneficiarían del tratamiento farmacológico, recordando que las medidas no farmacológicas como la actividad física, la dieta y la cesación tabáquica están recomendadas para todas las mujeres, y que la prevención de caídas es también importante en las mujeres mayores.

Entonces, parece lógico y atractivo plantear la decisión de iniciar un tratamiento farmacológico destinado a la prevención de las fracturas osteoporóticas (antiresortivos, terapia de reemplazo hormonal, calcitonina, raloxifeno, etc.) basándose en la estimación individual del riesgo absoluto de fracturas.

Sin embargo y dado que esta herramienta para la toma de decisiones diagnósticas y terapéuticas fue desarrollada sobre los datos la población española y con el objetivo de ser aplicada localmente, habrá que considerar la adaptación de modelos como éste al medio donde nos desempeñamos.

En este sentido vale resaltar la sencilla regla de predicción desarrollada por Ciapponi y col. ${ }^{11} \mathrm{y}$ validada sobre dos muestras no probabilísticas de mujeres mayores de 45 años de Buenos Aires, Argentina.

Esta regla, que requiere contar con la información sobre la edad y el peso de las pacientes, permite identificar con un $95 \%$ de sensibilidad y un valor predictivo negativo de $98 \%$ a las mujeres con OP femoral. Ver tablas 6 y 7 .

Queda también como tarea pendiente la evaluación de los resultados consecutivos a la utilización de este tipo de herramientas respecto de su impacto en la reducción de fracturas osteoporóticas.

Tabla 6: regla de predicción de osteoporosis densitométrica femoral con dos variables desarrollada en Buenos Aires (Argentina) por Ciapponi y col.

\begin{tabular}{|c|c|c|c|}
\hline \multicolumn{2}{|c|}{ Variable clínica } & $\begin{array}{c}\text { Asociación con osteoporosis } \\
\text { densitométrica OR (LG95\%) }\end{array}$ & Puntaje \\
\hline \multirow[t]{4}{*}{ 1. Edad } & 45 a 54 años & 1 & 0 \\
\hline & 55 a 64 años & $3,58(1,92$ а 10,75$)$ & 4 \\
\hline & 65 a 74 años & $7,29(2,37$ a 22,42$)$ & 7 \\
\hline & Más de 75 años & $18,10(0,80$ a 6,43$)$ & 18 \\
\hline \multirow[t]{3}{*}{ 2. Peso } & Más de $70 \mathrm{~kg}$ & 1 & 0 \\
\hline & 60 a $69 \mathrm{~kg}$ & $2,33(0,92$ a 5,85$)$ & 2 \\
\hline & Menos de $60 \mathrm{~kg}$ & $7,03(3,00$ a 16,44$)$ & 7 \\
\hline
\end{tabular}

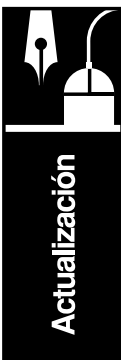

Tabla 7: riesgo predicho para osteoporosis femoral de acuerdo a la regla de Ciapponi y col.

\begin{tabular}{c|c|c} 
Puntaje & Riesgo aproximado de osteoporosis & $\begin{array}{c}\text { Indicación de } \\
\text { densitometría ósea }\end{array}$ \\
\hline $\mathbf{3}$ ó menos & $<1,5 \%$ & No \\
\hline $\mathbf{4}$ & 1,5 a $2,5 \%$ & \\
\hline $\mathbf{5}$ a $\mathbf{9}$ & 4 a $9,9 \%$ & \multirow{2}{*}{ Si } \\
\hline $\mathbf{1 1}$ a $\mathbf{1 3}$ & 10 a $15 \%$ & \\
\hline $\mathbf{1 4}$ a $\mathbf{2 0}$ & 20 a $25 \%$ & \\
\hline $\mathbf{2 0}$ ó más & Mayor a $25 \%$ & \\
\hline
\end{tabular}


Bibliografía

1. National Osteoporosis Foundation.America's bone health: the state of osteoporosis and low bone mass in our nation. Washington (DC): National Osteoporosis Foundation; 2002.

2. Ettinger MP. Aging bone and osteoporosis: strategies for preventing fractures in the elderly. Arch Intern Med. 2003 Oct 13;163(18):2237-46.

3. Seeman E. Osteoporosis: trials and tribulations. Am J Med. 1997 Aug 18;103(2A):74S-87S; discussion 87S-89S.

4. North American Menopause Society. Management of osteoporosis in postmenopausal women: 2006 position statement of The North American Menopause Society. Menopause. 2006 May-Jun;13(3):340-67; quiz 368-9

5. Barrett-Connor E, y col. Osteoporosis and fracture risk in women of different ethnic groups. J Bone Miner Res. 2005 Feb;20(2):185-94. Epub 2004 Oct 18.

6. Miller PD, Siris ES, Barrett-Connor E, Faulkner KG, Wehren LE, Abbott TA, Chen YT, Berger ML, Santora AC, Sherwood LM. Prediction of fracture risk in postmenopausal white women with peripheral bone densitometry: evidence from the National Osteoporosis Risk Assessment. J Bone Miner Res. 2002 Dec;17(12):2222-30.

7. Siris ES, Brenneman SK, Barrett-Connor E, Miller PD, Sajjan S, Berger ML, Chen YT. The effect of age and bone mineral density on the absolute, excess, and relative risk of fracture in postmenopausal women aged 50-99: results from the National Osteoporosis Risk Assessment (NORA). Osteoporos Int. 2006;17(4):565-74. Epub 2006 Jan 4.

8. Czerwiński E, Badurski JE, Marcinowska-Suchowierska E, Osieleniec J. Current understanding of osteoporosis according to the position of the World Health Organization (WHO) and International Osteoporosis Foundation. Ortop Traumatol Rehabil. 2007 Jul-Aug;9(4):337-56.

9. Dirección General de Farmacia y Productos Sanitarios. Recomendaciones para la valoración y tratamiento de la osteoporosis primaria en mujeres de la Comunidad de Madrid. Madrid: Comunidad de Madrid, Consejería de Sanidad; 2007. Disponible en url: http://www.infodoctor.org/rafabravo/rec-osteo.pdf. Ultimo acceso 25/03/2008.

10. Kanis JA, Johnell O, De Laet C, Jonsson B, Oden A, Ogelsby. International variations in hip fracture probabilities: implications for risk assessment. J Bone Miner Res. 2002; 17:1237-44.

11. Ciapponi A y col. Regla de Predicción de Osteoporosis en Buenos Aires (PROBA): Desarrollo y Validación. Archivos de Medicina Familiar y General. Vol 5(1) 2007: 5-14 Disponible en URL: http://www.famfyg.org.ar/revista/revista_famfyg/volumen4_n1/inv_originales01.pdf (último acceso 04/04/08).

\section{INFO - EVIDENCIA}

\section{¿Qué es Evidencia, Actualización en la Práctica Ambulatoria?}

Una publicación independiente editada cada dos meses desde 1997 por la Fundación MF, organización sin fines de lucro dedicada a promover el desarrollo de la medicina familiar y la atención primaria de la salud.

\section{¿Cuál es el objetivo de Evidencia?}

Contribuir a la educación continua y a la actualización de los profesionales de la salud de la región en el área de la atención ambulatoria.

\section{Contenidos de Evidencia}

- Información independiente, actualizada y resumida en forma sencilla y en castellano sobre temas relevantes de la atención ambulatoria.

- Artículos seleccionados por su calidad y relevancia clínica, resumidos y comentados críticamente por destacados profesionales del área.

- Revisiones sobre temas clínicos, epidemiológicos, sanitarios o humanísticos, de importancia para la práctica ambulatoria.

- Notas farmacológicas para la actualización rápida de temas de terapéutica clínica.

- Discusión de casos clínicos con herramientas de Medicina Basada en la Evidencia.

- Glosario de términos de epidemiología y medicina basada en la evidencia

¿Cómo es posible acceder a Evidencia?

Por suscripción a la edición en papel, o bien por Internet, en nuestro sitio: http://www.evidencia.org 